\title{
Hyperacusis in patients with complex regional pain syndrome related dystonia
}

\author{
M J M de Klaver, M A van Rijn, J Marinus, W Soede, J A P M de Laat, J J van Hilten
}

See end of article for

authors' affiliations

J Neurol Neurosurg Psychiatry 2007;78:1310-1313. doi: 10.1136/jnnp.2006.111609

.....................

Correspondence to:

Dr J J van Hilten, Department

of Neurology, Leiden

University Medical Centre,

PO Box 9600, NL-2300 RC

Leiden, the Netherlands;

J.J.van_Hilten@lumc.nl

Received 22 November 2006

Revised 11 April 2007

Accepted 16 April 2007

Published Online First

30 April 2007

\begin{abstract}
Introduction: In complex regional pain syndrome type 1 (CRPS-1), patients may have manifestations of central involvement, including allodynia, hyperalgesia or dystonia. We noted that more severely affected patients may experience hyperacusis, which may also reflect central involvement. The aim of this study was to evaluate the occurrence and characteristics of hyperacusis in patients with CRPS related dystonia.

Methods: The presence of hyperacusis, speech reception thresholds (SRT), pure-tone thresholds (PTT) and uncomfortable loudness (UCL) was evaluated in 40 patients with CRPS related dystonia.

Results: PTT and SRT were normal for all patients. 15 patients (38\%) reported hyperacusis and this was associated with allodynia/hyperalgesia and with more affected extremities. UCLs of patients with hyperacusis were significantly lower than UCLs of patients without hyperacusis.

Conclusion: Hyperacusis is common among severely affected patients with CRPS related dystonia and may indicate that the disease spreads beyond those circuits related to sensory-motor processing of extremities.
\end{abstract}

$\mathrm{C}$ omplex regional pain syndrome (CRPS) is frequently preceded by a trauma $(70-90 \%)$. In the acute phase, the clinical presentation is dominated by various combinations of sensory and autonomic symptoms and signs. ${ }^{12}$ Some patients with chronic CRPS may also develop movement disorders such as tremor, myoclonia and dystonia. ${ }^{3}$

Because of our clinic's special interest in movement disorders and CRPS, we had the opportunity to evaluate the more severely affected patients in whom CRPS evolved into a disabling disorder with prominent dystonia of multiple extremities. In the course of these evaluations, we noted that some patients reported hyperacusis ${ }^{4}$ (ie, an intolerance of ordinary sound levels). Hyperacusis is primarily associated with painful sensations to sound, which eventually may result in avoidance-like behaviour, whereas phonophobia is an anxious sensitivity towards specific sounds, largely independent of its volume. ${ }^{5}$ Contrary to phonophobia, hyperacusis is not directly related to fear of sound. ${ }^{6}$ Hyperacusis can arise from damage to the inner ear and eighth nerve, but has also been associated with central nervous system involvement as may occur in migraine. $^{67}$

In CRPS, patients may experience an increased response to a painful stimulus (hyperalgesia) or even pain when the skin is gently touched (allodynia). Both sensory features have been associated with abnormal excitability of nociceptive neurons within the central nervous system, a process known as central sensitisation. ${ }^{8}$ Pathophysiological studies in CRPS have provided evidence of functional changes at different levels of the central nervous system..$^{9}$

Taken together, the increased sensitivity to ordinary sound levels in patients with CRPS may suggest that this is yet another manifestation of central involvement in this disorder. Against this background we evaluated the occurrence and characteristics of hyperacusis in patients with CRPS related dystonia.

\section{METHODS}

\section{Patients}

Patients with a diagnosis of CRPS and dystonia in one or more extremities who were referred to our department for treatment of dystonia between January 2000 and May 2006 were included in this study. Patients were generally referred from pain clinics and from departments of anaesthesiology, rehabilitation medicine and surgery. Patients had to meet the CRPS diagnostic criteria of the International Association for the Study of Pain. ${ }^{11}$ According to these criteria, patients must have (1) continuing pain, allodynia or hyperalgesia, in which the pain is disproportionate to any inciting event, (2) evidence at some time of oedema, changes in skin blood flow or abnormal sudomotor activity in the region of the pain and (3) no condition that would otherwise account for the degree of pain and dysfunction. Exclusion criteria were other disorders that could cause auditory impairment. The study protocol was approved by the hospital ethics committee and all patients gave informed consent.

\section{Audiogram}

Pure-tone audiogram thresholds (PTT), uncomfortable loudness (UCL) and speech reception thresholds (SRT) were assessed with an ENT audiometer by a certified audiologist using a standard method. ${ }^{12}{ }^{13}$ Briefly, for determination of PTT, patients wearing a headphone had to press a button when they heard a tone in the frequency range $250-8000 \mathrm{~Hz}$ that was presented at $5 \mathrm{~dB}$ increments (within the range 0-120 dB). To establish the patient's UCL, tones were presented in a similar manner as for determination of PTT and patients had to indicate when they considered the sound level as uncomfortably loud. SRT was determined using the standard consonantvowel-consonant word list on CD (prerecorded female speaker) of the Dutch Society of Audiology. ${ }^{14}$ All words were balanced on an rms level, sublists were homogeneous with regard to speech reception scores and normative values were available. Each list consisted of equivalent sublists of 11 Dutch three-phoneme monosyllables. Based on the individual PTT, tests were done at a fixed presentation level around the PTT. The first list of words was always presented at a level of $+20 \mathrm{~dB}$ above the threshold.

Abbreviations: CRPS, complex regional pain syndrome; $\mathrm{FI}$, Fletcher Index; PCCL, Pain Coping and Cognition List; PIT, pure-tone threshold; SRT, speech reception threshold; UCL, uncomfortable loudness 
Table 1 Demographic data and complex regional pain syndrome characteristics of the patients

\begin{tabular}{|c|c|}
\hline No of patients & 40 \\
\hline $\operatorname{Sex}(F / M)$ & $38 / 2$ \\
\hline Age (y) (mean (SD)) & $41.9(10.2)$ \\
\hline Duration of complaints (y) (mean (SD)) & $11.4(7.5)$ \\
\hline \multicolumn{2}{|l|}{ Type of onset } \\
\hline Unknown & 11 \\
\hline Contusion & 10 \\
\hline Fracture & 10 \\
\hline Operation or IV & 9 \\
\hline \multicolumn{2}{|l|}{ Location of onset } \\
\hline Upper extremity (L/R) & $6 / 9$ \\
\hline Lower extremities $(L / R)$ & $9 / 16$ \\
\hline \multicolumn{2}{|l|}{ No of affected extremities } \\
\hline 1 & 2 \\
\hline 2 & 7 \\
\hline 3 & 12 \\
\hline 4 & 19 \\
\hline \multicolumn{2}{|l|}{ Spreading pattern } \\
\hline None & 2 \\
\hline Ipsilateral & 22 \\
\hline Heterolateral & 12 \\
\hline Diagonal & 4 \\
\hline VAS pain $(0-100 \mathrm{~mm})($ mean (SD)) & $71.4(16.3)$ \\
\hline \multicolumn{2}{|l|}{ Movement disorders } \\
\hline Dystonia & 21 \\
\hline Dystonia + tremor & 6 \\
\hline Dystonia + myoclonia & 11 \\
\hline Dystonia + tremor + myoclonia & 2 \\
\hline \multicolumn{2}{|l|}{ Allodynia and/or hyperalgesia } \\
\hline Yes & 25 \\
\hline No & 15 \\
\hline \multicolumn{2}{|l|}{ Hyperacusis } \\
\hline Yes & 15 \\
\hline No & 25 \\
\hline
\end{tabular}

For most subjects this results in $100 \%$ phoneme score. Afterwards, lists are presented at levels in decreasing steps of $10 \mathrm{~dB}$ until the subject can hardly understand the tokens and reaches a score below $50 \%$. The threshold is then determined by simple linear interpolation of the percentages found for the levels just above and below 50\%.

For PTT and UCL values, a low Fletcher Index (FI-low: mean over the frequency range 500-2000 Hz) and a high Fletcher Index (FIhigh: mean over frequency range 1000-4000 Hz) were calculated. In general, normal values for the SRT and PTT do not exceed $20 \mathrm{~dB}^{12}$ A UCL threshold of $100 \mathrm{~dB}$ is considered normal and values below $100 \mathrm{~dB}$ indicate the presence of hyperacusis. ${ }^{13}$

\section{Clinical characteristics}

Demographic and clinical information was collected and included pain intensity, number of affected extremities, type of motor impairments, presence of allodynia or hyperalgesia and presence of hyperacusis. In addition, the Pain Coping and Cognition List (PCCL) was administered. ${ }^{15}$ The PCCL includes a subscale on pain catastrophising ( 12 items), which was used to assess the potential relation between a more focused attention to external stimuli and hyperacusis.

\section{Data analysis and statistics}

Data were analysed with SPSS 12.01 (SPSS Inc., 2003), using parametric tests for normally distributed continuous data and non-parametric tests for other data. Pearson's correlation coefficient was used to compare SRT, PTT and UCL between both ears of each patient. The significance threshold was set at $\mathrm{p}<0.05$.

\section{RESULTS}

Demographic information and CRPS characteristics of the 40 patients are presented in table 1. Table 2 shows the differences in CRPS characteristics between patients with and without hyperacusis.

As the correlations of SRTs, PTTs and UCLs (both for FI-low and FI-high) between the right and left ear of each patient were high ( all $>0.7 ; p<0.001$ ), we used the mean thresholds of both ears in the subsequent analyses. SRT and the PTT (FI-low and FI-high) for all patients were within the normal range (table 3 ). The mean UCL for both FI-low and FI-high were significantly lower $(\mathrm{p}<0.001$ for both thresholds) in our patient group compared with the normal population value of $100 \mathrm{~dB}$.

Patients with hyperacusis had significantly lower UCLs at all of the indicated frequencies compared with patients without hyperacusis (fig 1). Disease duration did not differ significantly between patients with hyperacusis (13.1 years) compared with patients without hyperacusis $(10.4$ years; $p=0.365)$. Seven of the 15 patients with hyperacusis reported tinnitus.

Thirty-one patients had three or four affected extremities of which 15 reported hyperacusis. Interestingly, none of the nine patients with one or two affected extremities reported hyperacusis (Fisher's exact test, $\mathrm{p}=0.015$ ). However, patients with one or two affected extremities did not differ significantly in UCL thresholds (FI-high or FI-low) from patients with three or four affected extremities.

Patients without hyperalgesia and/or allodynia less frequently reported hyperacusis compared with patients with these sensory symptoms $\left(\chi^{2} ; p=0.026\right)$. The odds ratio for hyperacusis in patients with hyperalgesia/allodynia was 7.0 (95\% CI 1.7 to 12.4). The UCLs did not differ significantly for both FI-low and Fl-high between patients with hyperalgesia/ allodynia and patients without these symptoms.

Patients with hyperacusis had lower scores on the pain catastrophising subscale of the PCCL ( 2.5 vs $3.2 ; \mathrm{p}<0.05)$.

\section{DISCUSSION}

Although our findings are limited to an extreme phenotype, to the best of our knowledge this is the first study to evaluate hyperacusis in CRPS. Thirty-eight per cent of patients with CRPS related dystonia in our study reported hyperacusis, whereas the prevalence of hyperacusis in the general population is less than $2 \% .{ }^{16}$ Auditory function, evaluated by means of the PTT and SRT, showed no differences between patients and the general population. UCLs of CRPS patients not experiencing hyperacusis were normal. In contrast, UCLs of patients with hyperacusis were significantly lower than UCLs of patients without hyperacusis. It is unlikely that the presence of hyperacusis is explained by a more focused attention to external stimuli as in such cases higher scores on pain catastrophising would have been expected in patients with hyperacusis compared with patients without hyperacusis but, surprisingly, the opposite was found.

Interestingly, patients with hyperacusis more often experienced allodynia and/or hyperalgesia, which are manifestations of central sensitisation. This phenomenon concerns the increased sensitivity of spinal neurons, despite a lack of change of afferent input. ${ }^{17}$ Patients with hyperacusis also had more extremities with dystonia, which is associated with central disinhibition. ${ }^{18} 19$ The degree of spread of dystonia, therefore, likely reflects a marker of severity of central involvement. Although the difference was not significant, patients with hyperacusis had a mean duration of disease of 2.7 years longer than those without hyperacusis, which may suggest the possibility that with further progression of the disease, some of the patients without hyperacusis in this study ultimately would develop hyperacusis. Taken together, the sensory and motor features of this phenotype provide circumstantial evidence that hyperacusis in these patients is initiated centrally. The high correlations of SRTs, PTTs and UCLs between both 
Table 2 Differences between patients with and without hyperacusis

\begin{tabular}{llll}
\hline & No hyperacusis & Hyperacusis & p Value \\
\hline No of patients & 25 & 15 & \\
Sex (F/M)* & $25 / 0$ & $13 / 2$ & 0.061 \\
Age (y)t (mean (SD)) & $42.7(9.6)$ & $40.6(11.3)$ & 0.538 \\
Disease duration (y)† (mean (SD)) & $10.4(4.9)$ & $13.1(10.5)$ & 0.365 \\
Type of onset & 8 & 3 & \\
Unknown & 7 & 3 & \\
Contusion & 4 & 6 & \\
Fracture & 6 & 3 & \\
Operation or IV & & & \\
Location of onset & $3 / 5$ & $3 / 4$ & \\
Upper extremity (L/R) & $4 / 13$ & $0 / 8$ & \\
Lower extremities (L/R) & 2 & & \\
No of affected extremities & 7 & 0 & \\
1 & 6 & 0 & \\
2 & 10 & 6 & \\
3 & 2 & 9 & \\
4 & 12 & 0 & \\
Spreading pattern & 9 & 10 & \\
None & 2 & 3 & \\
Ipsilateral & & & \\
Heterolateral & $68.7(16.1)$ & $75.4(16.4)$ & \\
Diagonal & & & \\
VAS pain (O-100 mm)† (mean (SD)) & 15 & 6 & \\
Movement disorders & 5 & 1 & \\
Dystonia & 4 & 7 & \\
Dystonia + tremor & 1 & 1 & \\
Dystonia + myoclonia & 13 & & \\
Dystonia + tremor + myoclonia & 12 & & \\
Allodynia and/or hyperalgesia* & & & \\
Yes & & & \\
No & & & \\
\hline VAS, Visual Analogue Scale. & & & \\
*the $\chi^{2}$ test; tthe t test for independent samples. & & & \\
\end{tabular}

ears in each patient make an unilateral peripheral cause unlikely and further support the central involvement in hyperacusis. However, the question remains how the pathophysiology of hyperacusis and the central features of CRPS interact.

Key to central sensitisation is the disturbed inhibitoryexcitatory balance, which is associated with multiple biological changes in the central nervous system. These biological changes may include increased activity in excitatory pathways where substance $\mathrm{P}$, excitatory neurotransmitters and adenosine triphosphate act via voltage gated calcium channels and/or diminished activity in inhibitory pathways via gamma-aminobutyric acid and glycine. ${ }^{20}$ Interestingly, these neurotransmitters and neuropeptides not only play a role in synaptic transmission of the auditory system but also act as tropic agents that modulate auditory signal processing as a result of sensory experience. ${ }^{21} 22$ By altering auditory type I neural excitability to glutamate, these neuropeptides, for example, could induce hyperacusis and contribute to the induction, maintenance or exacerbation of tinnitus in the auditory periphery. $^{23}$

\begin{tabular}{lcc}
\multicolumn{2}{l}{ Table 3} & Threshold values \\
\hline Measurement & Mean value (dB) & $95 \% \mathrm{Cl}$ \\
\hline PTI FI-Low & 12.1 & $8.9-15.4$ \\
PTT FI-High & 15.5 & $11.4 .-19.5$ \\
SRT & 10.3 & $7.7-12.9$ \\
UCL FI-Low & 79.9 & $71.4-88.3$ \\
UCL FI-High & 78.9 & $70.4-87.5$
\end{tabular}

Fl-low/high, low/high Fletcher Index; PTT, pure-tone threshold; SRT, speech reception threshold; UCL, uncomfortable loudness.

Values are means and $95 \% \mathrm{Cl}$ of the thresholds for all 40 patients.
In CRPS, central sensitisation may spread in an ipsilateral somatotopic distribution up the neuraxis to involve nociceptive processing at the level of the thalamus or higher cortical centres. ${ }^{24}$ Because different sensory inputs converge at the level of the thalamus, central sensitisation may affect auditory circuitry. On the other hand, hyperacusis in our patients was related to the perception of discomfort, and not to the sound perception threshold. Hence the "annoyance factor" of hyper-

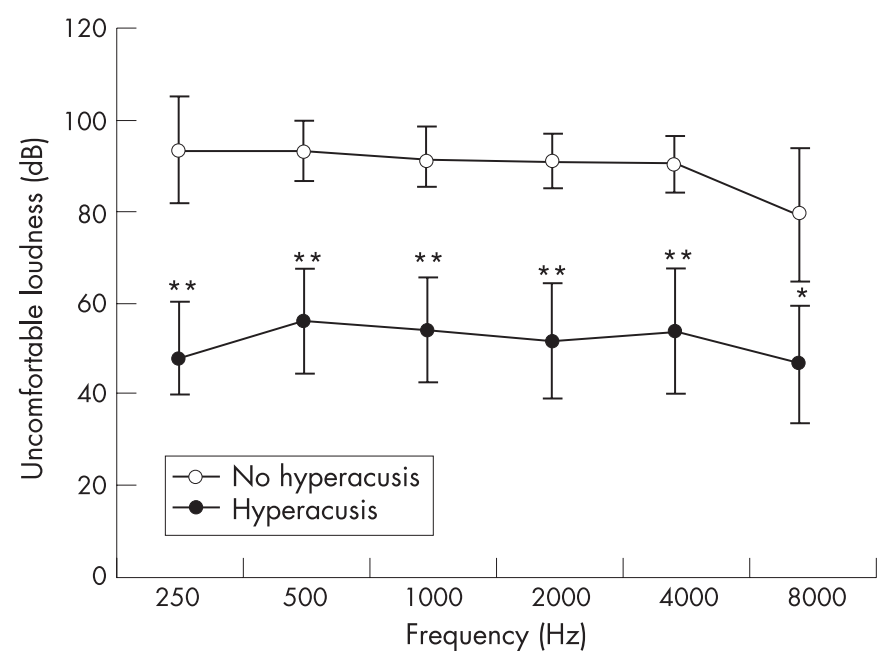

Figure 1 Uncomfortable loudness levels (with 95\% Cl) in patients with complex regional pain syndrome, with $(n=15)$ and without $(n=25)$ hyperacusis. ${ }^{*} p<0.05,{ }^{* *} p<0.001$ compared with patients without hyperacusis. High and low Fletcher Index values for patients with hyperacusis (52.6 dB and $53.8 \mathrm{~dB}$, respectively) were significantly lower $(p<0.001)$ compared with the high and low Fletcher Index values of patients without hyperacusis $(91.0 \mathrm{~dB}$ and $91.7 \mathrm{~dB}$, respectively). 
acusis may indicate a role of limbic activation, as has been implicated for other features of CRPS and tinnitus. ${ }^{25-28}$ A potential limitation of this study is that the patients reflect an extreme phenotype of CRPS, limiting any conclusion regarding the prevalence of hyperacusis in CRPS patients in general. The association between hyperacusis and dystonia could have been evaluated more thoroughly if data regarding the occurrence of hyperacusis in severely affected patients without dystonia had been available. It is also important to note that the assessment technique of evaluating UCL thresholds relies on patient provided information, rendering the findings sensitive to subjective influences. Our findings may stimulate the development of objective assessment techniques that aim to evaluate manifestations of central sensitisation in the auditory system.

In conclusion, we found that hyperacusis is common among severely affected patients with CRPS related dystonia. Hyperacusis in these patients may reflect the spreading of central sensitisation to auditory circuitry.

\section{ACKNOWLEDGEMENTS}

We thank Professor JHM Frijns for his contribution to this manuscript. This study was performed within TREND (Trauma Related Neural Dysfunction), a knowledge consortium that integrates research on Complex Regional Pain Syndrome type 1.

\section{Authors' affiliations}

M J M de Klaver, M A van Rijn, J Marinus, J J van Hilten, Department of Neurology, Leiden University Medical Centre, Leiden, the Netherlands W Soede, J A P M de Laat, Audiological Centre, Leiden University Medical Centre Leiden, the Netherlands

Funding: The project was supported by a grant from the Dutch Government (BSIK03016).

Competing interests: None.

\section{REFERENCES}

1 Allen G, Galer BS, Schwartz L. Epidemiology of complex regional pain syndrome: a retrospective chart review of 134 patients. Pain 1999;80:539-44.

2 Veldman PH, Reynen HM, Arntz IE, et al. Signs and symptoms of reflex sympathetic dystrophy: prospective study of 829 patients. Lancet 1993;342:1012-16.

3 van Hilten JJ, Blumberg H, Factor IV. Movement disorders and dystrophypathofiology and measurements. In: Wilson PR, Stanton-Hicks MS, Harden RN, eds. CRPS: current diagnosis and therapie. Seattle: IASP Press, 2005:1 19-37.

4 van Hilten JJ, van de Beek WJ, Hoff JI, et al. Intrathecal baclofen for the treatment of dystonia in patients with reflex sympathetic dystrophy. N Engl J Med 2000;343:625-30.
5 Schaaf $\mathbf{H}$, Klofat B, Hesse G. Hyperacusis, phonophobia, and recruitment. Abnormal deviations of hearing associated with hypersensitivity to sound. HNO 2003;51:1005-11.

6 Katzenell U, Segal S. Hyperacusis: review and clinical guidelines. Otol Neurotol $2001 ; 22: 321-6$.

7 Gordon AG. "Hyperacusis" and origins of lowered sound tolerance. J Neuropsychiatry Clin Neurosci 2000;12:117-19.

$8 \mathrm{Ji}$ RR, Woolf CJ. Neuronal plasticity and signal transduction in nociceptive neurons: implications for the initiation and maintenance of pathological pain. Neurobiol Dis 2001;8:1-10.

9 Fukumoto M, Ushida T, Zinchuk VS, et al. Contralateral thalamic perfusion in patients with reflex sympathetic dystrophy syndrome. Lancet 1999;354:1790-1.

10 Maihofner C, Handwerker HO, Neundorfer B, et al. Patterns of cortical reorganization in complex regional pain syndrome. Neurology 2003;61:1707-15.

11 Merskey H, Merskey H, Bogduk N. Classification of chronic pain: Description of chronic pain syndromes and definitions of pain terms, 2nd Edn. Seattle, IASP Press, 2006:40-3.

12 Man A, Naggan L, Bergman M. Classification of the severity of acoustic trauma based on pure tone threshold audiometry. Acta Otolaryngol 1981;92:25-31.

13 Sherlock LP, Formby C. Estimates of loudness, loudness discomfort, and the auditory dynamic range: normative estimates, comparison of procedures, and test-retest reliability. J Am Acad Audiol 2005; 16:85-100.

14 Bosman AJ, Smoorenburg GF. Intelligibility of Dutch CVC syllables and sentences for listeners with normal hearing and with three types of hearing impairment. Audiology 1995;34:260-84.

15 Stomp-van den Berg SGM, Vlaeyen JWS, Ter Kuile MM, et al. Pïn coping en cognitie lijst (PCCL). Maastricht: Pijn Kennis Centrum, Academisch Ziekenhuis Maastricht, 2001.

16 Sammeth CA, Preces DA, Brandy WT. Hyperacusis: Case studies and evaluation of electronic loudness suppression devices as a treatment protocol. Scan Audiol 2000;29:28-36.

17 Woolf CJ. Dissecting out mechanisms responsible for peripheral neuropathic pain: implications for diagnosis and therapy. Life Sci 2004;74:2605-10.

18 Schwenkreis $\mathbf{P}$, Janssen $\mathrm{F}$, Rommel $\mathrm{O}$, et al. Bilateral motor cortex disinhibition in complex regional pain syndrome (CRPS) type I of the hand. Neurology 2003:61:515-19.

19 van de Beek WJ, Vein A, Hilgevoord AA, et al. Neurophysiologic aspects of patients with generalized or multifocal tonic dystonia of reflex sympathetic dystrophy. J Clin Neurophysiol 2002;19:77-83.

20 Woolf CJ, Mannion RJ. Neuropathic pain: aetiology, symptoms, mechanisms, and management. Lancet 1999;353:1959-64.

21 Hunter C, Doi K, Wenthold RJ. Neurotransmission in the auditory system. Otolaryngol Clin North Am 1992;25:1027-52.

22 Pujol R, Puel JL, Eybalin M. Implication of non-NMDA and NMDA receptors in cochlear ischemia. Neuroreport 1992;3:299-302.

23 Sahley TL, Nodar RH, Musiek FE. Endogenous dynorphins: possible role in peripheral tinnitus. Int Tinnitus J 1999:5:76-91.

24 Drummond PD, Finch PM. Sensory changes in the forehead of patients with complex regional pain syndrome. Pain 2006;123:83-9.

25 Drummond PD, Finch PM, Skipworth S, et al. Pain increases during sympathetic arousal in patients with complex regional pain syndrome. Neurology $2001 ; 57: 1296-303$

26 Jastreboff PJ, Gray WC, Gold SL. Neurophysiological approach to tinnitus patients. Am J Otol 1996;17:236-40.

27 Vuilleumier P. How brains beware: neural mechanisms of emotional attention. Trends Cogn Sci 2005;9:585-94.

28 Wall PD. The mechanisms by which tissue damage and pain are related-an updated review. In: Campbell JN, eds. Pain. Seattle: IASP press, 1996:123-6. 